\title{
Hydrodynamic Forces and Band Formation in Swimming Magnetotactic Bacteria
}

\author{
D. C. Guell $\|$, H. Brenner \|, R. B. Frankel I and H. Hartman * \\ Department of Chemical Engineering $\|$, Francis Bitter National Magnet \\ Laboratory Il, and Department of Earth, Atmospheric and Planetary Sciences*, \\ Massachusetts Institute of Technology, Cambridge, Massachusetts 02139, U.S.A.
}

\begin{abstract}
Dense suspensions of magnetotactic bacteria form long, thin, stable bands perpendicular to the common, net direction of motion of the cells in a magnetic field. A mechanism for this phenomenon is proposed in which the hydrodynamic coupling between two swimming cells is described in terms of an approximate, far-field solution for the flow around one swimming cell. The calculated hydrodynamic interaction force and torque based upon this analysis are of the correct orders of magnitude and in the right directions to provide a reasonable explanation for band formation and stability.
\end{abstract}

\section{Introduction}

Magnetotactic bacteria from aquatic sediments migrate along magnetic field lines (Blakemore, 1982; Frankel, 1984). The magnetotactic response of these organisms is due to intracytoplasmic $\mathrm{Fe}_{3} \mathrm{O}_{4}$ particles, the magnetosomes (Balkwill et al., 1980) that constitute a fixed, permanent magnetic dipole in each cell. The dipole is oriented more-or-less parallel to the axis of motility of the cell. Alignment of the magnetic dipole in the direction of the local magnetic field as the cell swims causes the cell to move along magnetic field lines. Thus, the cell is effectively a seif-propelled magnetic compass needle.

Magnetotactic bacteria propel themselves in their aquatic environment by rotating their flagellum(ae) (Berg, 1975). In this regard they are similar to all free-swimming bacteria, such as Escherichia coli (Macnab \& Aizawa, 1984; Berg, 1975). However, $E$. coli and other chemotactic bacteria exhibit a characteristic "run and tumble" motility that is due to reversals of the direction of rotation of the flagellum(ae) (Larsen et al., 1974). Counter-clockwise rotation causes the cell to travel in a relatively straight line, whereas clockwise rotation causes the cell to tumble. Magnetotactic 
bacteria with unidirectional motility ${ }^{\prime}$ do not tumble, but continue to swim along magnetic field lines for periods up to one hour or more. As such, it may be inferred that they never reverse the direction of flagellum rotation.

Spormann (1987) and Carlile et al. (1987) have recently reported an unusual migration phenomenon in dense suspensions of unidirectional magnetotactic bacteria swimming in narrow bore glass tubes when subjected to magnetic fields. Instead of the bacteria traveling down the tube in a cloud that dispersed with time owing to the continuous distribution of swimming speeds in the population, the cells formed stable, migrating waves or bands that translated with their long axes perpendicular to their common direction of motion, as shown in Fig. 1. The number of bacteria in a band was variable, up to several thousand, and cell separations generally approximated five body diameters. Small "protobands" or lateral strings, consisting of as few as two or three cells, were also observed. Cells within a given band migrated at the same speed, but different bands moved at different speeds; that is, band formation was accompanied by velocity sorting of the bacteria in the population. Cells within a given band exhibited complex local motions, in addition to which their positions within the band appeared to change continually; yet the band itself remained stable for relatively long times (Spormann, 1987). Reversing the direction of the magnetic field in the tube caused the bands to break up, subsequently reform, and migrate in the opposite direction, with the faster bands, initially behind the slower ones, catching up and eventually moving to the fore.

Using vertically- and horizontally-oriented Helmholtz coil pairs, Spormann found that several conditions were required for band formation:

(i) cells had to be highly motile;

(ii) both horizontal (parallel to the axis of the tube) and vertical magnetic field components were simultaneously required;

(iii) the horizontal field component had to be greater than a threshold value of $\sim 0.8$ gauss;

(iv) the vertical field component had to be greater than a threshold value of $\sim 0.2$ gauss.

Reducing either the vertical or horizontal magnetic field component below these threshold values caused break up of the bands.

Carlile et al. (1987) also observed band formation. They speculated that band formation resulted from magnetic interactions between the permanent, magnetic dipole moments of the bacteria as the latter swim. However, Spormann calculated the magnetic dipole moments of his bacteria $\left(\sim 3 \times 10^{-13} \mathrm{emu}\right)$ by estimating the $\mathrm{Fe}_{3} \mathrm{O}_{4}$ content of the cells from electron micrographs. He noted that for cells in close proximity, the dipole-dipole interaction energy can be attractive and larger

$\dagger$ Most magnetotactic cells found in sediments are unidirectional swimmers, migrating only either parallel or antiparallel to the local magnetic field direction. However, some bacteria, notably Aquaspiriilum. magnetofacticum (Blakemore, 1982) and other magnetotactic spirilla (Spormann \& Wolfe, 1984) are bipolarly flagellated, permitting them to swim in either direction along the magnetic field lines, as well as allowing them to spontaneously reverse direction. Band formation has been observed only for unidirectional swimmers (Spormann, 1987). 


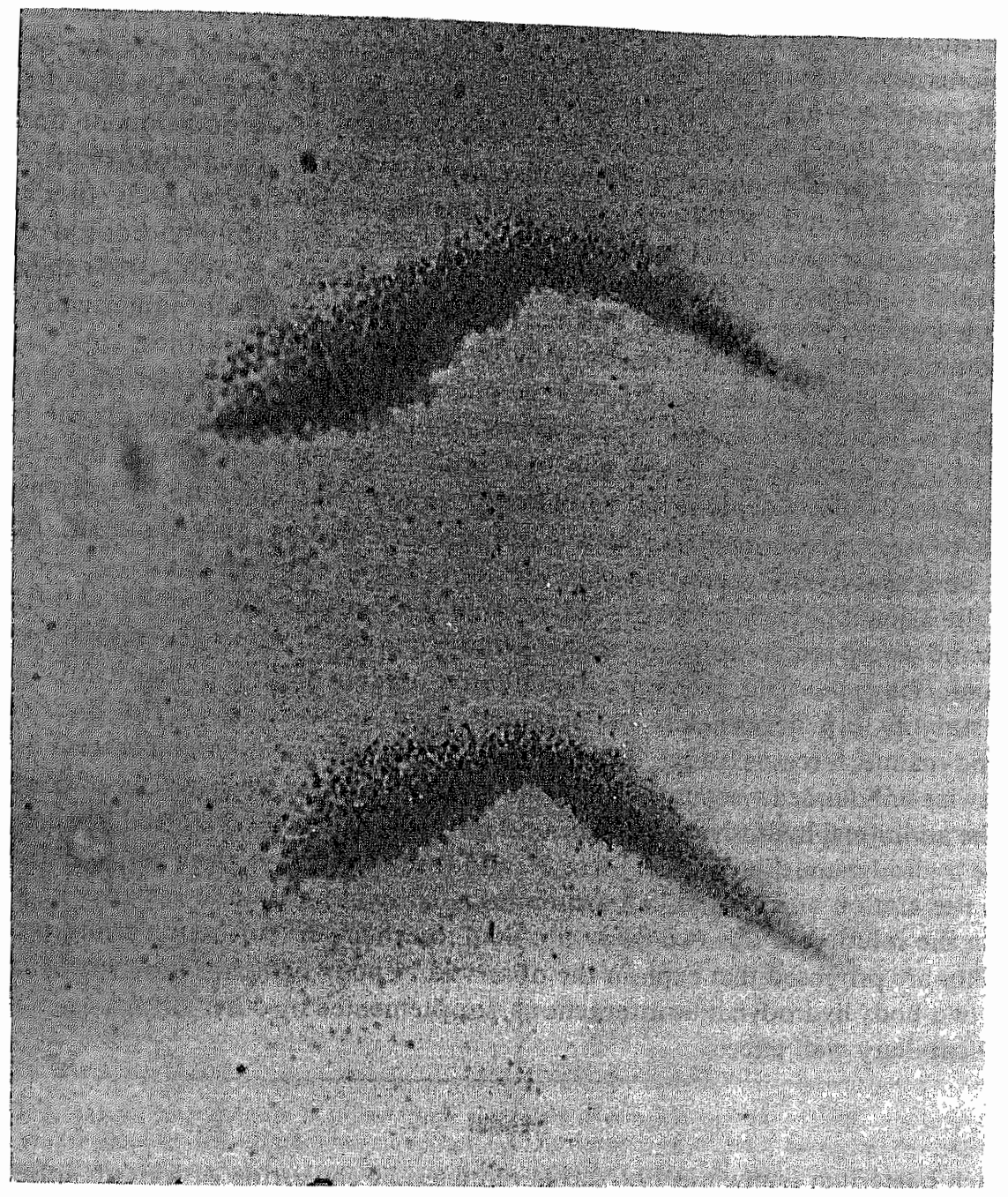

FIG. 1. Photograph showing that long, thin bands of bacteria form and move perpendicular to their long axes in the presence of an externally applied magnetic field.

than $k_{\mathrm{B}} T$ (the thermal or Brownian energy), but only for cells in close, longitudinal (head-to-tail) contact. For cells in lateral contact, the magnetic interaction is repulsive. He speculated that band formation was due to hydrodynamic coupling between cells swimming parallel to each other.

Band formation also occurs in populations of chemotactic and aerotactic bacteria. However, models for this behavior (Keller \& Segel, 1971) generally involve interaction of the individual cells with the chemical gradient(s), and do not consider interactions between cells. Band-like formations also occur in dense populations of 
spermatozoa (Rothschild, 1949). Much work has been done to analyze the motion of microscopic organisms [see, for example, the reviews by Jahn \& Votta (1972) and Brennen \& Winet (1977)]. Briefly, Taylor (1951a,b), Hancock (1953) and Gray \& Hancock (1955), in their classic papers, considered the propulsion of flexible cylinders or planes propagating plane displacement waves. Batchelor (1970), Cox (1970) and Lighthill (1976) expanded the usefulness of slender-body theory in problems of microbiological propulsion. Blake $(1972,1974)$ applied both slenderbody and fundamental hydrodynamic singularity (Chwang \& Wu, 1975) theories to problems involving ciliated organisms. Higdon (1979) modeled (numerically) the motion of swimming cells by superposing hydrodynamic singularities.

This paper demonstrates that hydrodynamic coupling between bacteria swimming parallel to each other results in a lateral attraction that could account for the band formation reported by Spormann and by Carlile et al. We propose that the role of the magnetic field is twofold: (i) to concentrate the cells, enabling hydrodynamic forces to overcome Brownian and cell-cell magnetic forces; and (ii) to orient the cells, such that hydrodynamic forces may act in a concerted manner.

While a quantitatively accurate simulation of the motion of the individual bacteria in a band would require solution of an $N$-body, non-linear, time-dependent, hydrodynamic/magnetic field problem, a qualitative understanding of band formation and stability can nevertheless be obtained from an analysis of the quasistatic hydrodynamic forces existing between two proximate swimming bacteria in an otherwise unbounded viscous fluid. Specifically, we address the problem of computing the additional hydrodynamic force and torque (respectively referred to hereafter as the hydrodynamic interaction force $\mathrm{F}^{+}$and torque $\mathrm{T}^{+}$) on a given cell which arise from the motion of a second cell swimming nearby in an infinite expanse of fluid. Given the solution of this two-body problem, mechanisms for band formation and stability are proposed that explain the observed N-body behavior in terms of many such two-body hydrodynamic interactions, supplemented by other effects, e.g. singlebody capillary wall effects.

\section{Model}

Each bacterium (shown in Fig. 2a) is modeled as consisting of a rigid, spherical body (or head) of radius $a$, to which is attached a rigid, helical flagellum that rotates, thereby providing propulsion for the entire organism. The flagellum thickness is $2 w$, its radius of curvature $b$, effective length $s$ (as measured along the centerline), and projected length $l_{2}$. The flagellum is supposed attached to the body by a straight segment of length $l_{1}$ (of negligible hydrodynamic resistance), furnishing a total fore-aft length $L$. We assume the body to be sufficiently distant from the operational portion of the flagellum $\left(a / l_{1} \ll 1\right)$ such that the two do not interact hydrodynamically. We further assume that: (i) the flagellum is much longer than it is thick; (ii) its thickness is much less than its radius of curvature; and (iii) its radius of curvature is much less than the cell's total length. These are quantified by the respective inequalities

$$
w / s \ll 1, \quad w / b \ll 1, \quad b / L \ll 1 . \quad(1 \mathrm{a}, \mathrm{b}, \mathrm{c})
$$




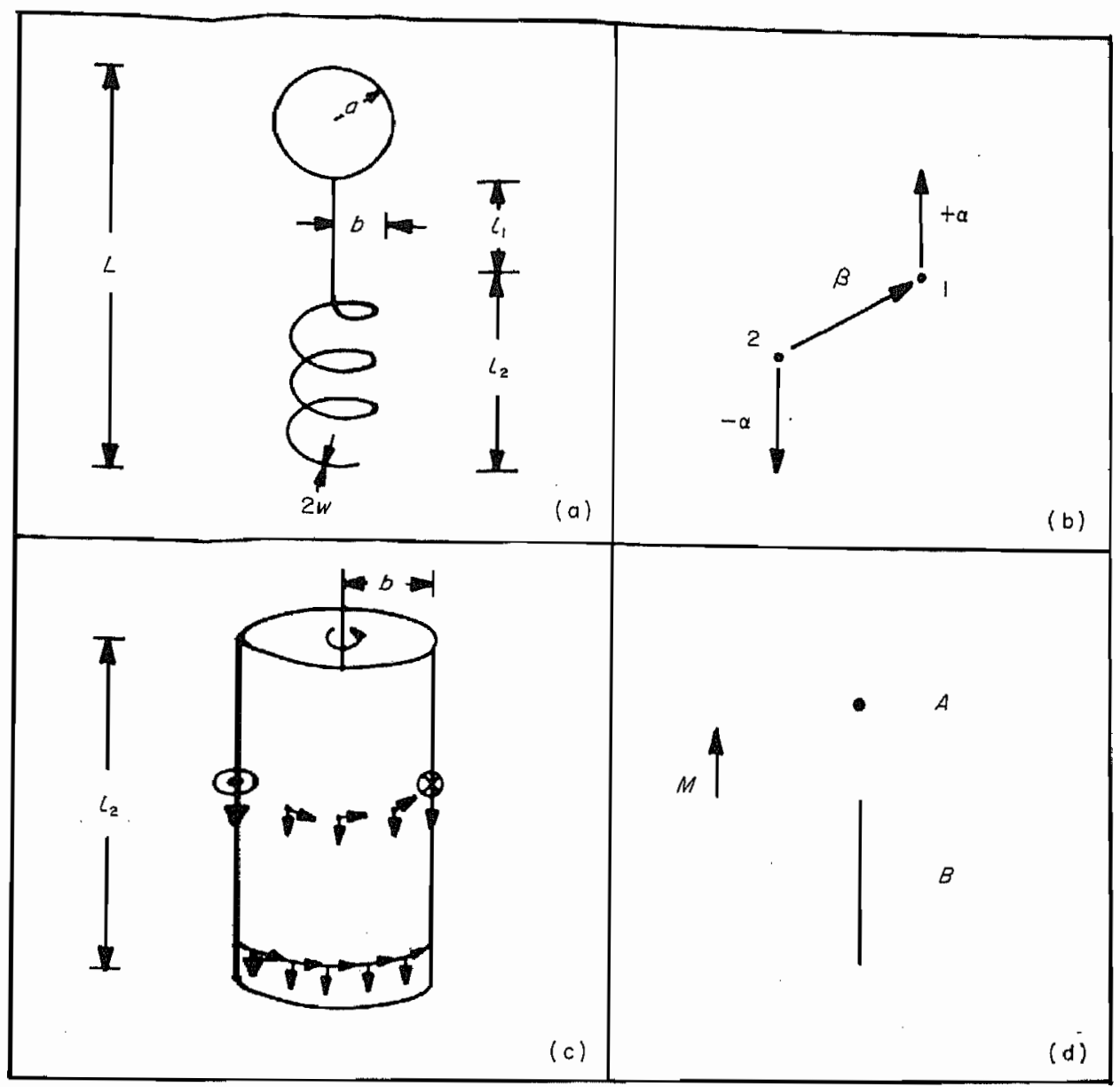

FIG. 2. (a) Schematic figure of a bacterium consisting of a spherical body or head and a flagellum; (b) A stokes-doublet derived from two stokeslets; (c) Distribution of stokeslets on the circular cylindrical envelope representing a time-average flagellum; (d) Simplified singularity distribution representing one swimming cell. ( $M$ denotes the direction of motion, $A$ the discrete stokeslet and couplet representing the body, and $B$ the $\mathbf{u}$ niform continuous distribution of stokeslets and couplets representing the fiagellum.)

Additionally, the body is assumed to contain a permanent magnetic dipole, which is aligned along the helical axis of the flagellum.

The fluid velocity and pressure fields $(\mathbf{v}, p)$ are governed by the well-known quasistatic Stokes' and continuity equations (Happel \& Brenner, 1983),

$$
\nabla^{2} \mathbf{v}=\frac{1}{\mu} \nabla p, \quad \nabla \cdot \mathbf{v}=0,
$$

which are appropriate for small Reynolds number flows. Translational and rotational Reynolds numbers based upon the sizes and relevant speeds of the body and 
flagellum are respectively

$$
\operatorname{Re}_{B}=\frac{\rho U a}{\mu}=7 \times 10^{-5}, \quad \operatorname{Re}_{F}=\frac{\rho w^{2} \omega_{F}}{\mu}=4 \times 10^{-8},
$$

with $U$ the characteristic cell velocity through the fluid and $\omega_{F}$ the magnitude of the angular velocity with which the flagellum rotates about its own axis; in the preceding, $\mu$ and $\rho$ are respectively the carrier fluid viscosity and density. Furthermore,

$$
\frac{\operatorname{Re}_{F}}{\mathrm{Sr}}=\left(\frac{a \omega_{F}}{U}\right) \operatorname{Re}_{F}=2 \times 10^{-8},
$$

with Sr the Strouhal number (expressing the ratio of intrinsic to imposed time scales). The small magnitude of (4) confirms that a quasistatic analysis will prove sufficiently accurate for present purposes. Thus, time derivative terms, $\rho \mathrm{dv} / \partial t$, do not appear in the momentum eqn (2a); accordingly, time $t$ plays the role of a parameter rather than that of an independent variable.

\section{Theory}

Because it does not easily lend itself to conventional boundary-value approaches (Happel \& Brenner, 1983), the low Reynolds number hydrodynamic problem posed will be solved (approximately) by using a superposition of fundamental hydrodynamic singularities. The usefulness of such methods in resolving microbiological hydrodynamic problems has been amply demonstrated (Brennen \& Winet, 1977). While singularity superposition methods have been used extensively to analyze the locomotion of single organisms, we believe this to be their first application to problems of hydrodynamic interaction between two self-propelling microorganisms.

Hydrodynamic singularities constitute exact solutions of the Stokes' and continuity eqns (2) in an unbounded fluid. They furnish the respective hydrodynamic velocity and pressure fields arising from the action of some singular dynamical or kinematical mode, such as an isolated force, couple or source acting at a point of the fluid. The "stokeslet" represents the hydrodynamic field due to an isolated external point force acting on the fluid. Its single vector parameter $\boldsymbol{\alpha}$ expresses the magnitude and direction of this vector force via the equation

$$
\mathbf{F}=8 \pi \mu \boldsymbol{\alpha} .
$$

Likewise, a "couplet" arises from an external point couple exerted on the fluid. A "potential doublet" (or "source doublet") arises from the residual limit of a fiuid source and sink of equal strength in close proximity.

The "stokes-doublet" arises from the superposition of two equal and opposite stokeslets in the limit as their separation distance vanishes while their strength goes to infinity (in such a way that the resulting fluid velocity and pressure fields remain of order unity). This singularity is particularly important in expressing the far-field effect of swimming cells, such as those under consideration. In Fig. 2b, the properties of a stokes-doublet are characterized by two vector parameters, $\boldsymbol{\alpha}$ and $\boldsymbol{\beta}$. Parameters 
$\alpha$ and $-\boldsymbol{\alpha}$ denote the respective strengths of stokeslets 1 and 2 composing the stokes-doublet, whereas $\boldsymbol{\beta}$ is the vector drawn from stokeslet 2 to 1 .

Whereas subsequent hydrodynamic calculations pertain to cells in an unbounded viscous fluid, the actual experiments involved cells swimming near a capillary wall. Existence of the wall seems necessary for band formation, since it provides a barrier allowing cells to become sufficiently close to interact. However, the concomitant hydrodynamic wall effect upon cell motion is not the essential element inducing band formation; in particular, neither the velocity nor pressure fields arising from the hydrodynamic interaction of a cell with the wall possess characteristics capable of causing individual cells to combine into bands. Accordingly, hydrodynamic wall effects will be neglected in the subsequent analysis. Errors resuiting therefrom are estimated in the accompanying Appendix.

\section{HYDRODYNAMICS OF THE BODY AND FLAGELLUM}

Body and flagellum hydrodynamics will be separately considered since hydrodynamic interactions between these two cell constituents are assumed negligible. The spherical head or body of the cell will be regarded as translating and rotating in the infinite fluid at velocity $U$ (with respect to the quiescent fluid at infinity) and frequency $\omega_{B}$ in response to the respective "external" force and torque exerted on it by the flagellum. The solution of this linear problem consists of a superposition of the classical solutions of Stokes for a translating sphere (cf. Brennen \& Winet, 1977) and Kirchoff for a rotating sphere (cf. Chwang \& Wu, 1974), and is accordingly expressible as the sum of a stokeslet, potential doublet and couplet, each placed at the sphere center. The stokeslet parameter $\boldsymbol{\alpha}$ for the translating sphere is

$$
\boldsymbol{\alpha}=\frac{3}{4} a \mathbf{U} .
$$

Values of the comparable couplet and potential doublet parameters need not be explicitly assigned at the level of approximation for which our result eventually proves valid.

The flagellum is modeled as a rigid helix which rotates, providing propulsion (Berg, 1975) for the cell as a whole, in the manner of a screw propeller driving a ship; strictly speaking, this analogy may be misleading since ship propulsion occurs at very large, rather than very small, Reynolds numbers. Clearly, hydrodynamic effects due to the flagellum motion are complex; but they can be made tractable using slender-body theory (Lighthill, 1976). For bodies satisfying the dual inequalities

$$
r_{0} / L_{0} \ll 1, \quad r_{0} / R_{0} \ll 1,
$$

with $r_{0}, L_{0}$ and $R_{0}$ respectively, a characteristic radius, length (as measured along the centerline) and radius of curvature, slender-body theory reveals that the hydrodynamic effect of a body moving at small Reynolds number can be calculated solely in terms of a distribution of stokeslets and potential doublets placed along the centerline of the body. 
Upon supposing (for simplicity) the the magnitude of the force exerted by the fluid on the flagellum is uniform along its length, the requisite singularity distribution can be found from the analysis of Schreiner (1971), who computed the force on each element of a rigid rotating helix in an unbounded quiescent viscous fluid. This reveals that the force exerted by the fluid upon each element of the helix may be decomposed into a "thrust" component, directed parallel to the helical axis and in the direction of cell motion, and a "torque" component, directed normal to both the radial vector drawn from the axis of rotation through the element and to the direction of cell motion. It is this latter component that produces the torque required to turn the helix.

As the flagellum turns, each element rotates about the helix axis, thereby creating a time-periodic fluid-mechanical effect. However, since the long-time behavior of the cells is of primary interest, we assume (as confirmed in the Appendix) that the time scale on which the flagellum rotates is much less than that characterizing changes in the mean separation distance between adjacent cells. This allows the instantaneous singularity distribution representing the flagellum to be replaced by an average distribution over the surface swept out by the helical elements during one complete turn.

During one such revolution, each element of the helix traces out a circle as the helix turns, whence the latter sweeps out a circular cylinder of radius $b$. Thus, to the order of our approximation, the instantaneous stokeslet distribution along the helix is replaced with a stokeslet distribution over this cylindrical envelope. At each point on this cylindrical surface is placed a stokeslet of the same strength and direction as would exist at that point at the moment it is intersected by a helix element. The stokeslet distribution on this envelope possesses two components: (i) a uniform component, antiparallel to the direction in which the organism moves, and (ii) a second uniform component, directed tangentially. This distribution is pictured in Fig. 2c.

At large distances ( $b / r \ll 1$, with $r$ the distance from the cell), the thrust stokeslet distribution over the cylinder appears as a uniform distribution of stokeslets along the helical axis, provided that $b / l_{2} \ll 1$. This far-field approximation introduces an error of $O\left(\alpha b / r^{2}\right)$, where $\alpha$ is the magnitude of a stokeslet equivalent to the total thrust force. Furthermore, at large distances the "torque" stokeslet distribution appears as a uniform distribution of couplets along the axis, at least to $O\left(r^{-2}\right)$.

The leading-order effect of the motion of a swimming cell is that of the (cancelling) stokeslet distribution, which is respectively of orders $O\left(r^{-2}\right)$ and $O\left(r^{-3}\right)$ in the velocity and pressure fields far from the body. Since the velocity and pressure fields of a potential doublet decay as $O\left(r^{-3}\right)$ and $O\left(r^{-4}\right)$ respectively, the distribution of potential doublets can be neglected to leading order. Figure $2 \mathrm{~d}$ shows the resulting approximate distribution of singularities representing the solution of Stokes equations around one swimming cell.

Once the cell axis is aligned along the magnetic field, no net torque is exerted on the fluid, whence the respective far-field velocity and pressure fields arising from the couplet distribution, being $O\left(r^{-3}\right)$ and $O\left(r^{-4}\right)$, can be neglected. Insofar as leading-order effects far $(L / r \ll 1)$ from the body are concerned, the stokeslet 
distribution may be replaced by a stokes-doublet. Thus, the hydrodynamic field arising from a single cell is expressible solely as a stokes-doublet characterized by the parametric values

$$
\boldsymbol{\alpha}=\hat{\mathbf{u}}_{4}^{\frac{3}{3}} a U, \quad \boldsymbol{\beta}=\hat{\mathbf{u}} l^{*} . \quad(8 \mathrm{a}, \mathrm{b})
$$

Here, $\hat{\mathbf{u}} \equiv \mathbf{U} /|\mathbf{U}|$ denotes a unit vector in the direction of the cell's motion; additionally, $l^{*}=(1 / 2)\left(L+l_{2}\right)$, whereas $U \equiv|\mathrm{U}|>0$ is the cell's swimming speed.

With the effect of the cell motion expressed in this singular fashion, approximate expressions for the velocity and pressure fields existing in a hypothetical unbounded fluid surrounding a single swimming bacterium can be computed in a co-ordinate system at rest with respect to the fluid at infinity, and with its origin chosen to instantaneously coincide with the cell-body center at that moment. These fields are respectively those arising from a stokes-doublet possessing the stated parameters; explicitly

$$
\begin{aligned}
& \mathbf{v}(\mathbf{x})=\hat{\mathbf{x}}_{4}^{3} U \frac{l^{*}}{a}\left(\frac{a}{r}\right)^{2}\left[3 \cos ^{2}(\hat{\mathbf{u}}, \hat{\mathbf{x}})-1\right], \\
& p(\mathbf{x})=-\frac{3}{2}\left(\frac{\mu U}{a}\right) \frac{l^{*}}{a}\left(\frac{a}{r}\right)^{3} \sin ^{2}(\hat{\mathbf{u}}, \hat{\mathbf{x}}),
\end{aligned}
$$

with $\mathbf{x}$ a position vector drawn from the cell center, $\hat{\mathbf{x}}=\mathbf{x} /|\mathbf{x}|$ a unit position vector, and $(\hat{\mathbf{u}}, \hat{\mathbf{x}}) \equiv \cos ^{-1}(\hat{\mathbf{u}} . \hat{\mathbf{x}})$ the angle between $\hat{\mathbf{u}}$ and $\hat{\mathbf{x}}$. Recall that in simplifying the thrust stokeslet distribution, an error of magnitude $O\left(\alpha b / r^{2}\right)$ was incurred in the velocity. The ratio of this error to the computed effect is

$$
b / l^{*}<2 b / L \ll 1,
$$

which was assumed small at the outset.

Our computation of the velocity and pressure fields generated by a single cell moving through an unbounded fluid permits the concomitant forces and torques exerted on a second cell present in that flow to be approximated by applying the method of reflections (Happel \& Brenner, 1983). This method constitutes an analytic perturbation scheme for deriving convergent series solutions of zero Reynolds number hydrodynamic, multibody, no-slip, boundary-value problems. In short, if two relatively distant cells are spaced many characteristic body sizes apart, the force on one cell arising from the motion of a second can be approximated by initially regarding the flow as extending to infinity with only the first cell present. This amounts to computing only the first term in the series, with all other terms being smaller than the first by a factor of $O(a / r)^{4}$.

The hydrodynamic interaction force and torque on a cell as a result of this flow can be computed by neglecting the additional force exerted on the flagellum (owing to its thinness), while retaining that exerted on the spherical head. The magnitude of the error incurred in doing so is estimated in the accompanying Appendix. Faxen's laws (Happel \& Brenner, 1983) then furnish the required hydrodynamic force $\mathbf{F}$ and torque $\mathbf{T}$ (the latter about the sphere center) exerted on a translating-rotating 
sphere immersed in an arbitrary unbounded Stokes flow as

$$
\mathbf{F}=6 \pi \mu a\left[-\mathbf{U}+\mathbf{v}_{0}^{\infty}+\frac{a^{2}}{6 \mu}\left(\nabla p^{\infty}\right)_{0}\right], \quad \mathbf{T}=8 \pi \mu a^{3}\left(\zeta_{0}^{\infty}-\omega\right) . \quad(11 \mathrm{a}, \mathrm{b})
$$

Here, $v^{\infty}, p^{\infty}$ and $\zeta^{\infty} \equiv(1 / 2) \nabla \times v^{\infty}$ are, respectively, the undisturbed, far-field velocity, pressure and vorticity fields, with the subscript " 0 " denoting evaluation of the field to which it is affixed at the (fluid) position currently occupied by the sphere center. The respective quantities

$$
\mathbf{F}^{+}=6 \pi \mu a\left[\mathbf{v}_{0}^{\infty}+\frac{a^{2}}{6 \mu}\left(\nabla p^{\infty}\right)_{0}\right], \quad \mathbf{T}^{+}=8 \pi \mu a^{3} \zeta_{0}^{\infty}
$$

represent the additional hydrodynamic force and torque arising from the motion of a second cell swimming nearby; that is, they constitute the hydrodynamic interaction force and torque. Explicitly, in present circumstances.

$$
\begin{aligned}
& \mathbf{F}^{+}=\hat{\mathbf{x}}_{2}^{9} \pi \mu a U \frac{l^{*}}{a}\left(\frac{a}{r}\right)^{2}\left[3 \cos ^{2}(\hat{\mathbf{u}}, \hat{\mathbf{x}})-1\right], \\
& \mathbf{T}^{+}=\hat{\mathbf{i}}^{*} 9 \pi \mu a^{2} U \frac{l^{*}}{a}\left(\frac{a}{r}\right)^{3} \sin 2(\hat{\mathbf{u}}, \hat{\mathbf{x}}),
\end{aligned}
$$

with $\hat{\mathbf{i}}^{*} \equiv \hat{\mathbf{u}} \times \hat{\mathbf{x}}$ a unit vector perpendicular to both $\hat{\mathbf{u}}$ and $\hat{\mathbf{x}}$.

These results show the hydrodynamic interaction force to be much greater than the magnetic cell-cell interaction forces, the (inverse) ratio being

$$
\frac{\left|\mathbf{F}_{\mathrm{mag}}\right|}{\left|\mathbf{F}^{+-}\right|}=1.6\left(\frac{a}{r}\right)^{2}
$$

This is obviously quite small at the experimentally observed $(a / r \approx 10)$ separation distances. Furthermore, comparison of hydrodynamic interaction forces with Brownian forces furnishes the force ratio

$$
\frac{\left|\mathbf{F}^{+}\right|}{\left|\mathbf{F}_{\mathrm{Br}}\right|}=270\left(\frac{a}{r}\right)^{2}
$$

Thus, for modest separation distances, the hydrodynamic interaction force exceeds the Brownian force by a wide margin. Even at 10 body radii separation, the hydrodynamic interaction force is still about three times that of the Brownian force, and hence sufficient to largely overcome the randomizing influence of such Brownian forces. Moreover, the time scale required for significant changes in cell separation arising from hydrodynamic interactions, computed from our result, is approximately $1 \mathrm{sec}-$ in good agreement with observation. We also find that the interaction torque can deflect cells from their "straight-ahead" orientation to the extent of up to $6^{\circ}$ for cells 10 body radii apart, and $18^{\circ}$ for 7 body radii.

The physical nature of these quantities is clarified in Figs $3 \mathrm{a}$ and $3 \mathrm{~b}$, which display the direction of $\mathrm{F}^{+}$and $\mathrm{T}^{+}$arising from a cell situated at the origin. Figure 3a clearly 


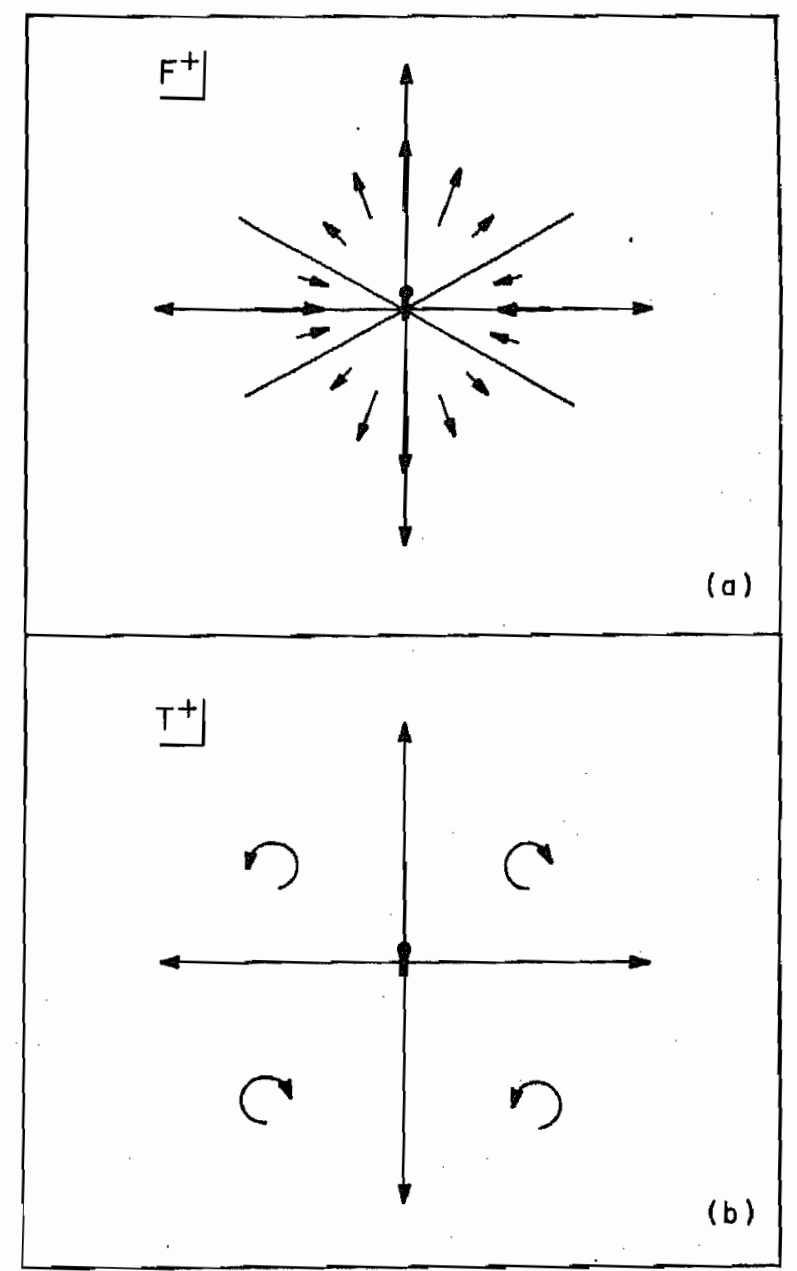

FIG. 3. Directions of interaction force $\mathrm{F}^{+}(\mathrm{a})$ and torque $\mathrm{T}^{+}(\mathrm{b})$ on a given cell situated near a second cell whose center lies at the origin. The direction of the arrow in (a) applies to the cell whose head (center) is situated at the point corresponding to the tail of the arrow; likewise, the indicated direction of rotation in (b) applies to the cell whose head (center) is situated at the point lying at the center of the indicated rotation circle. 
reveals the hydrodynamic interaction force to be attractive between two cells swimming side-by-side, and repulsive for head-to-tail swimmers.

\section{Discussion}

Given the preceding results for the two-body hydrodynamic problem, the following mechanisms are proposed to explain band formation and stability. In the inclined magnetic field and bacteria are constrained to move near the capillary wall-in close proximity to the other cells, and with all moving in very nearly the same direction. As a result of its self-propulsion each cell sets up a flow in the surrounding fluid, which manifests itself by exerting a force on its neighbors. This force attracts side-by-side neighbors and repels head-to-tail neighbors, causing cells to move into relative positions roughly side-by-side. Thereby, long, thin bands of bacteria form, with each band's long axis perpendicular to the common direction of net motion. Since bacterial populations display a distribution of swimming speeds, it is possible for a fast-swimming cell to swim past slower cells without being attracted to (and hence held within the band by) the latter cells. Thus, a faster cell can swim more-or-less freely until it encounters a band that moves at approximately that cell's own intrinsic speed.

The issue of band stability cannot, however, be explained solely in terms of the hydrodynamic forces arising from two-body interactions. This becomes evident upon posing two questions: (i) what keeps the fastest cell in a band from being expelled ahead of the band (and outrunning the latter) once that cell reaches the front of the band, where all its nearest neighbors are head-to-tail neighbors? and (ii) conversely, what keeps the slowest cell from being expelled behind the band (and hence being outrun by the latter) once that cell reaches the rear of the band?

The stability of the bands can be rationalized by invoking the following arguments. First, cells derive no mutual streamlining advantage from swimming together in pairs, as the average speed of a pair of identical cells swimming near to each other is identical to their individual swimming speeds in isolation. However, owing to hydrodynamic wall effects and deflection of cells from the "straight-ahead" direction (resulting from the hydrodynamic interaction torque), it is evident that the speed with which a band moves is less than the speed with which individual cells would move in isolation, far from the wall.

A fast cell rarely escapes from a stable band so as to move ahead of the band; rather, when such a cell arrives at the front of the band, the hydrodynamic interaction torque exerted on it tends to deflect it toward the closest end of the band, owing to the band shape (as depicted in Fig. 4a). The cell's velocity component in the direction of band motion is thus decreased and it does not outrun the band. Furthermore, the cell moves toward the end of the band, where the net hydrodynamic interaction force exerted on it by its neighbors tends to be attractive. Once at the side of the band the cell might then move toward the center, as cells near the center move forward within the band to replace those celis in front of them. This explains why slightly faster cells do not leave stable bands; moreover, it partially explains the "rolling" or continual exchange of cell positions within the band. 


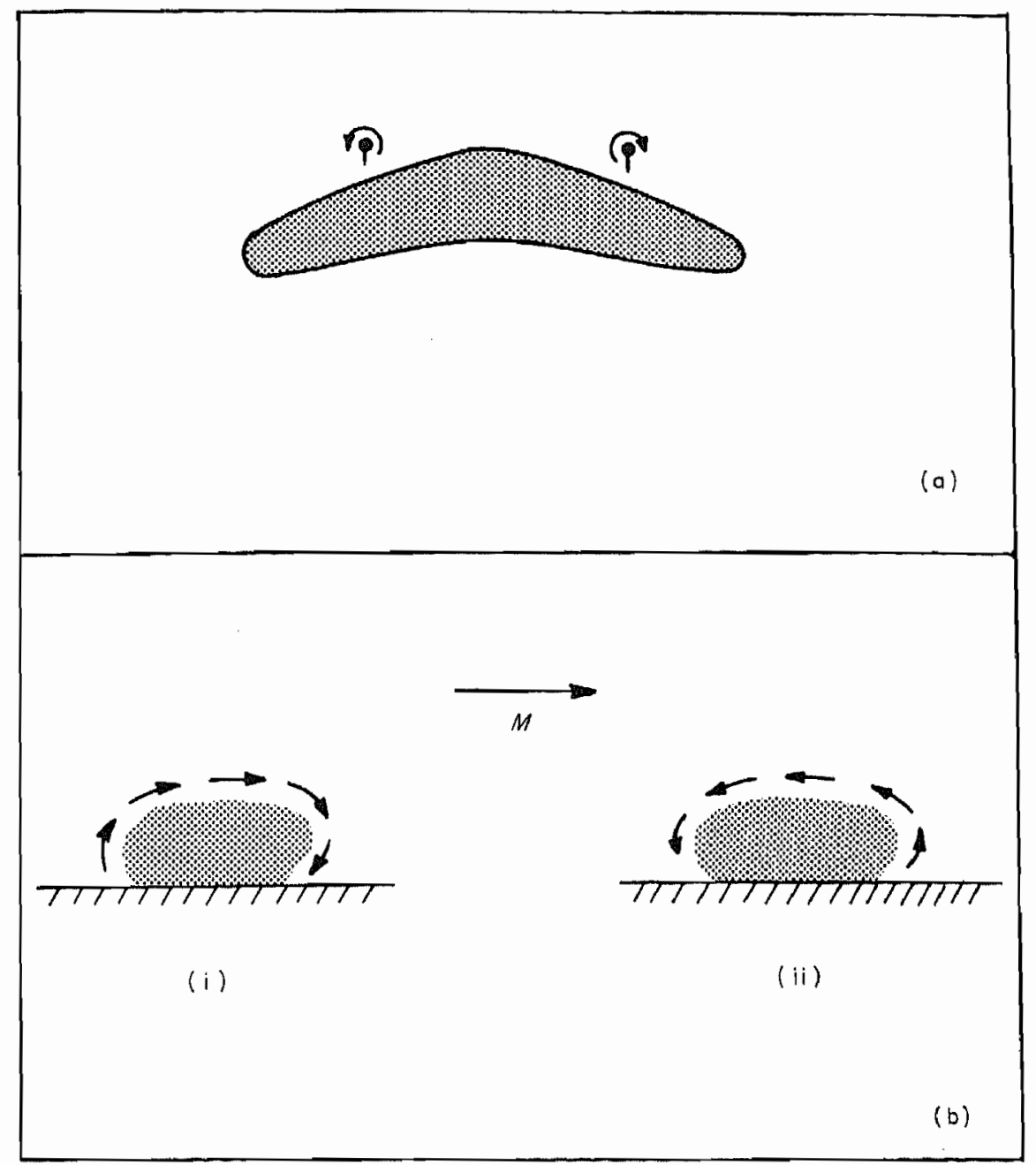

FIG. 4. (a) The net effect on the interaction torque deflects cells slightly ahead of a band toward the ends; (b) Side perspectives of two possible directions of band rolling. ( $M$ denotes the direction of band motion.)

The issue of why a band does not expel and subsequently outrun its slowest member once that cell arrives at the back of the band requires considering the other "component of rolling". Video tapes (Spormann, private communication) of the phenomenon reveal that in addition to moving "front-to-side-to-back-to-front", cells also roll from "front-to-back-to-front" by moving through positions above and below the band center.

Figure $4 \mathrm{~b}$ depicts possible side views of this motion. Since no video pictures exist from this perspective, it is not known whether the cells roll in mode (i) or (ii). However, direction (i) appears more likely, since those cells closer to the wall will 
move more slowly as a consequence of hydrodynamic wall effects. Assuming this to be the case, the rolling motion due to wall effects will cause cells at the rear of the band to move away from the wall, while those above move to the front. The slow cell will then be impeded less by wall effects when at the rear, enabling it to keep up with its own band. Though not furnishing a definitive mathematical proof of band stability, one that a more sophisticated analysis-such as an N-body, time-dependent, numerical simulation--might provide, such arguments nevertheless furnish a plausible qualitative explanation of the underlying physics contributing to band formation and stability.

\section{Conclusion}

This paper was motivated by an attempt to understand band formation in suspensions of magnetotactic bacteria swimming in the presence of an external magnetic field. The proposed explanation focuses on the hydrodynamic field which arises from the dual effects of a large cell body (which pushes fluid forward in the neighborhood of the forward parts of the swimming organism) and a helical flagellum (which pushes fluid aft along the tail region). Since these two force distributions are equilibrated, the stokes-flow result is roughly a stokes-doublet-consisting of a "dragging" stokeslet (representing the body) and a "thrusting" stokeslet (representing the flagellum). The resulting fluid-mechanical stresses generated by the fluid motion relative to the moving organism repels those neighbors situated forward and aft of it, but attracts those on its sides. Globally, this tends to arrange cells into side-by-side configurations, forming long, thin bands lying perpendicular to their common direction of net motion.

The issue of band stability is both more complex and subtle. It requires accounting for; (i) hydrodynamic wall effects, serving to slow individual cells and thereby contributing to rolling; (ii) hydrodynamic interaction torques, deflecting cells from their straight-ahead direction and hence also contributing to rolling; (iii) flow within the band created by the continual exchange of cell positions among neighbors.

Although hydrodynamic coupling of the type we have considered produces especially dramatic effects upon magnetotactic bacteria, it should be equally operative in dense suspensions of flagellar bacteria, regardless of the physical mechanism by means of which the preferred spatial direction arises. Indeed, band formation in chemotactic and aerotactic bacteria has long been known to occur (e.g. Beyerinck 1893; Adler, 1966a,b). However, band formation among such bacteria differs in kes respects from the phenomenon considered here. For instance, aerotactic bacteric typically give rise to no more than two bands (Adler, $1966 a, b$ ), whereas magnetotacti bacteria form many bands. Moreover, whereas such cells are directed by compley "thermodynamic" interactions with the chemical gradient, our magnetotactic bac teria are mechanically oriented by an external couple-an aspect wholly missing is these other cases. As such, previous explanations (Keller \& Segel, 1971) of banc formation generally focus on interaction of the individual cells with the chemica gradients, but neglect interactions between cells, opposite to the explanation of ban. formation offered here. 
Bands will not, however, necessarily form among all directed bacteria. For instance, if the bacterium's body is too small, no clear-cut separation will exist between the respective hydrodynamic thrust and drag forces. In this case, the demonstrated stokes-doublet velocity field imposed around a swimming bacterium will not constitute the correct leading-order effect, and the hydrodynamic interaction forces calculated here will vanish relative to other, more important hydrodynamic forces, which have been neglected. Additionally, unless the mechanism directing the bacteria can overcome the hydrodynamic interaction torque, the bacteria may tend to avoid one another under the influence of that torque. Thus, bands of such bacteria would prove unstable despite the continued importance of hydrodynamic interaction analyzed here. Finally, the biased random walk of chemotactic bacteria, with temporal reversals in the direction of flagellar rotation, could also prevent formation of stable bands. Nevertheless, hydrodynamic coupling might then result in local density fluctuations or correlated cellular motion within the suspension.

\section{Summary}

Dense suspensions of swimming bacteria embodying permanent magnetic dipoles are observed to form long, thin, stable bands perpendicular to the common direction of motion of the cells when subjected to a magnetic field possessing appropriate field-strength components. The magnetic field orients the cells such that they all swim in nearly the same direction. A mechanism is proposed in which hydrodynamic coupling between proximate cells accounts for the observed band formation and band stability phenomena.

Using singularity superposition methods, an approximate solution is obtained for the low Reynolds number fluid velocity and pressure fields around a single, isolated bacterium swimming in an unbounded viscous fluid. Approximate expressions are then obtained for the additional hydrodynamic interaction force and torque exerted on a second cell when it swims in the neighborhood of this first cell. This additional force and torque are each of the correct order-of-magnitude, and act in the proper direction to account for the observed phenomena. The interaction force is attractive for cell pairs swimming side-by-side, and repulsive for cells swimming head-to-tail. We argue that such a force tends to arrange cells into a side-by-side configuration. Furthermore, this hydrodynamic interaction force dominates competing Brownian and cell-cell magnetic forces. We also argue that the interaction torque contributes to band stability.

We thank A. M. Spormann for discussions and for providing a video tape of magnetotactic bacterial bands as well as the photograph shown in Fig. 1. R.B.F. was partially supported by the Office of Naval Research. The Francis Bitter National Magnet Laboratory is supported by the National Science Foundation. D.C.G. was supported by an NSF Graduate Fellowship, and H.B. by the Office of Basic Energy Sciences of the Department of Energy.

\section{REFERENCES}

ADLER, J. (1966a). Science, N.Y. 153, 708

ADLER, J. (1966b). J. Bact. 92, 121. 
Balkw1ll, D. L., Maratea, D. \& Blakemore, R. P. (1980). J. Bact. 141, 1399.

BATCHELOR, G. K. (1970), J. Fluid Mech. 44, 419.

Bero, H. C. (1975). Scient. Am. 233, 36.

Beyerinck, M. W. (1893). Zentbl. Bakt. Parasitkde 14, 827.

BLAKE, J. R. (1972). J. Fluid Mech. 55, 1.

BLAKE, J, R. (1974). J. Eng. Math. 8, 113.

BlAKE, J. R. \& ChWANG, A. T. (1974). J. Eng. Math. 8, 23.

Blakemore, R. P. (1982). A. Rev. Microbiol. 36, 217.

BrenNen, C. \& Winet, H. (1977). A. Rev. Fl. Mech, 9, 339.

Carlile, M., Dudeney, A. W. L., Hebenstreit, B. K. \& Heerema, R. H. (1987). J. Magn. Magn. Mater. 67, 291.

ChWANG, A. T. \& Wu, T. (1974), J. Fluid Mech. 63, 607.

CHWANG, A. T. \& Wu, T. (1975), J. Fluid Mech. 67, 787.

Cox, R. G. (1970). J. Fluid Mech. 44, 791.

FrANkEL, R. B. (1984). A. Rev. Biophys. Bioeng. 13, 85.

GrAY, J. \& HANCOCK, G. (1955). J. exp. Biol. 32, 802.

HANCOCK, G. J. (1953), Proc. $R$. Soc. A217, 96.

HAPPEL, J, \& BRENNer, H. (1983). Low Reynolds Number Hydrodynamics. Martinus Nijhoff: The Netherlands.

HIGDON, J. J. L. (1979). J. Fluid Mech. 90, 685.

JAHN, T. L. \& VOTTA, J. J. (1972). A. Rev. Fl. Mech. 4, 93.

KelleR, E. F. \& SEgEL, L. A. (1971). J. theor. Biol. 30, 235.

Larsen, S. H., Reader, R. W., Kort, N., Tso, W. W. \& Adler, J. (1974). Nature, Lond. $249,74$.

LIGHTHILL, J. (1976). SIAM Review 18, 161.

MacNaB, R. M. \& Aizawa, S. I. (1984), A. Rev, Biophys. Bioeng. 13, 51.

ROTHSCHILD, Lord (1949). Nature, Lond. 163, 358.

SCHREINER, K. E. (1971). J. Biomechanics 4, 73,

SPORMANN, A. M. \& WOLfE, R. S. (1984). FEMS Microbial Lett. 22, 171.

SPORMANN, A. M. (1987). FEMS Microbiol. Ecol. 45, 37.

TAYlor, G. I. (1951a). Proc. R. Soc. A209, 447.

TAYlor, G. 1. (1951 b). Proc. R. Soc. A211, 225 .

\section{APPENDIX A}

The most critical idealizations and simplifications made in (approximately) solving the cell-cell interaction problem are herein examined to demonstrate the correspondence between this idealized model and the prevailing physical circumstances.

Upon replacing the instantaneous flagellum singularity distribution by an average distribution over a cylindrical envelope, the time scale $T_{F}$ governing flagellum rotation was assumed much shorter than the time scale $T_{S}$ governing changes in cell separation. A characteristic flagellum frequency is $100 \mathrm{sec}^{-1}$, whence $T_{F}=$ $0.01 \mathrm{sec} . T_{S}$ represents the cell-cell separation distance divided by the relative velocity at which two cells approach one another as a consequence of their mutual hydrodynamic interaction. For cell pairs swimming side-by-side this velocity is twice the disturbance velocity created by a single cell swimming in isolation. The ratio of these time scales is thus

$$
\frac{T_{F}}{T_{S}}=\frac{3}{2}\left(\frac{U}{\omega_{F} a}\right) \frac{l^{*}}{a}\left(\frac{a}{r}\right)^{3}=14\left(\frac{a}{r}\right)^{3} .
$$

At 10 body radii separation, this ratio is 0.014 thereby justifying the assumption.

In Lighthill's slender-body theory the error incurred in approximating the singularity distribution along the flagellum by one of stokeslets and potential doublets along 
the center line is $O(w / s)$, while the error due to centerline curvature is $O(w / b)$. For the organisms considered here these parameters possess the approximate values

$$
w / s<0.003 \ll 1, \quad w / b \approx 0.02 \ll 1 .
$$

Neglect of hydrodynamic interaction between the spherical head and flagellum constituted a major simplification, one that would have proved entirely inappropriate had quantitative computations of individual cell swimming speeds been desired. However, such swimming speeds constitute an input to the model, and were assumed known a priori. Furthermore, hydrodynamic interactions between the head and flagellum can be shown to increase the magnitudes of both the hydrodynamic interaction force and torque for a specified swimming speed. Consequently, with respect to hydrodynamic interaction between body and flagellum, our result furnishes a lower bound.

Also ignored was the additional force on the flagellum resulting from hydrodynamic interactions with other bodies. Approximating the flagellum by a straight circular cylinder of length $l_{3}=l_{1}+l_{2}$ (for purposes of computing the order-ofmagnitude of the additional force on the flagellum) shows this additional force to be negligible compared with that exerted on the body whenever the following inequality [cf. Cox, 1970; eqns $(7 \cdot 10),(7 \cdot 12 b)]$ obtains:

$$
\frac{1}{3} \frac{l_{3}}{a}\left[\ln \left(\frac{l_{3}}{w}\right)-0 \cdot 81\right]^{-1} \ll 1 .
$$

For the bacterium in question, this parameter possesses a value of 0.48 , which is not strictly small compared with unity. The effect of the additional force on the fiagellum could be treated by superposing effects caused by fluid velocity components (normal and parallel to each element of the helical axis) deriving from motion of a nearby cell. However, incorporation of this detail would only obscure the elementary understanding of the phenomenon provided by the simple structure of eqns $(13 a, b)$. Since the additional force exerted on the flagellum would lie approximately in the direction of the flow (as with the spherical body), inclusion of this effect-while increasing the hydrodynamic interaction force-would change neither its order-ofmagnitude nor algebraic sign. Accordingly, our calculated result constitutes a lower bound with respect to the additional hydrodynamic force exerted on the flagellum.

Finally, whereas the important subsidiary role piayed by the capillary wall in band formation and stability was acknowledged, hydrodynamic wall effects were nevertheless systematically neglected. Inclusion of such hydrodynamic wall effects, at least to leading order, could have been accomplished by placing in mirror-image position (on the other side of a hypothetical flat wall) the appropriate image system (Blake \& Chwang, 1974) for each flow singularity. The far-field effect of a single cell is that of a stokes-doublet, and the leading-order effect of its image system (Blake \& Chwang, 1974) is that of a stokes-doublet of opposite strength. Thereby, an estimate can be obtained of the far-field effect upon a second cell swimming $r$ units away, with both cells situated $h$ units from the wall (Fig. 5). The ratio $R$ of the neglected to the incorporated effect is the square of the ratio of the intercell separation distance between cells 1 and 2 to the distance of the image point from 


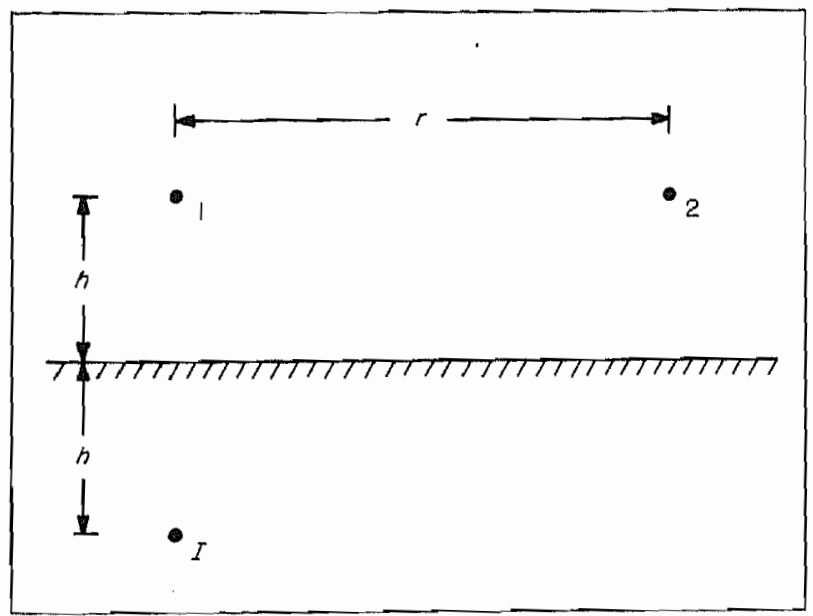

F1G. 5. The hydrodynamic wall effect on cell 2 is accounted for by placement of an image system $(I)$ arising from the presence of cell 1.

cell 2. Explicitly,

$$
R=\frac{r^{2}}{4 h^{2}+r^{2}}
$$

If $r / h \ll 1$, then

$$
R=\frac{r^{2}}{4 h^{2}} \ll 1,
$$

thereby rendering wall effects negligible. However, when $r / h \gg 1$, the results computed here are incorrect. In particular, it can be shown that hydrodynamic interaction forces are attenuated as $O\left(r^{-3}\right)$, rather than $O\left(r^{-2}\right)$. This decreases the order-ofmagnitude of the effect, as well as completely altering its direction.

For the intermediate case, $r / h \sim 1, R$ is still moderately small. For instance, $R=0.2$ and 0.1 respectively for $r / h=1$ and $2 / 3$. Again, since no photographs of bands are currently available from this perspective, there thus exist no measurements of characteristic cell-wall distances. Ignoring hydrodynamic wall effects clearly introduces some quantitative error, but even in the absence of the requisite data it is encouraging that wall effects would invalidate our qualitative explanation of the band formation phenomena only in the most extreme cases. 